\title{
Redundancy as a Measure of Fault-tolerance for the Internet of Things: A Review
}

\author{
Antonino Rullo ${ }^{1}$, Edoardo Serra ${ }^{2}$, and Jorge Lobo ${ }^{3 \star}$ \\ 1 DIMES Department, University of Calabria, 87036 Rende, Italy \\ n.rullo@dimes.unical.it \\ 2 Department of Computer Science, Boise State University, Boise, ID 83725, USA, \\ edoardoserra@boisestate.edu \\ 3 ICREA and Department of Information and Communication Technologies, \\ Universitat Pompeu Fabra, 08018 Barcelona, Spain \\ jorge.lobo@upf .edu
}

\begin{abstract}
In this paper we review and analyze redundancy-based faulttolerant techniques for the IoT as a paradigm to support two of the main goals of computer security: availability and integrity. We organized the presentation in terms of the three main tasks performed by the nodes of an IoT network: sensing, routing, and control. We first discuss how the implementation of fault-tolerance in the three areas is primary for the correct operation of an entire system. We provide an overview of the different approaches that have been used to address failures in sensing and routing. Control devices typically implement state machines that take decisions based on the measurement of sensors and may also ask actuators to execute actions. Traditionally state-machine replication for fault-tolerance is realized through consensus protocols. Most protocols were developed in the 80's and 90's. We will review the properties of such protocols in detail and discuss their limitations for the IoT. Since 2008, consensus algorithms took a new direction with the introduction of the concept of blockchain. Standard blockchain based protocols cannot be applied without modifications to support fault-tolerance in the IoT. We will review some recent results in this new class of algorithms, and show how they can provide the flexibility required to support fault-tolerance in control devices, and thus overcome some of the limitations of the traditional consensus protocols.
\end{abstract}

Keywords: Internet of Things · availability $\cdot$ redundancy $\cdot$ fault-tolerant · sensors $\cdot$ state machine $\cdot$ distributed consensus.

\section{Introduction}

In this paper we present a general overview at security in the Internet of Things (IoT) from the point of view of integrity and availability. In particular, we look at

\footnotetext{
* Jorge Lobo was partially supported by the Spanish Ministry of Economy and Com-
} petitiveness. Grant Numbers: TIN201681032P, MDM20150502. 
mechanisms to make an IoT network tolerant to component failures. Tolerance describes the ability of containing the effects of faults so that a system can avoid failures and continue working properly. Faults must be compensated in such a way that they not lead to a global system failure. The most common way to instrument fault-tolerance is through redundancy, i.e. the physical or logical replication of services. The intuition behind redundancy is that replication of the same service along multiple processing units confers the system the property of being resilient, that is, the ability to withstand the failure of some of its components when the number of healthy components far exceeds that of faulty ones. However, redundancy makes a system more complex and, consequently, more difficult to operate and manage.

Almost all fault-tolerant schemes proposed in the literature give systems the ability of re-configuring themselves to handle components failures and recoveries. Autonomic fault managements typically come in two flavors. In the first one, management is carried in two phases: a detection phase, where the faulty components are detected, and a re-configuration phase, where the faulty components are excluded and possibly replaced by healthy ones. The ability to agree on the state of the system allows the fault-free replicas to make correct and consistent progress. Alternatively, replicas go without identifying faulty components, as they operate by masking their effects. This management approach may be more efficient, as it does not need to perform additional detection and re-configuration tasks by giving up certainty. Implementations often rely on probabilistic methods, or only guarantee the computation of approximate results.

The implementation of fault-tolerance in the IoT may get more complicated when compared to traditional computer systems for many reasons. First, the IoT is characterized by high heterogeneity in many of its aspects: smart devices may differ from each other both in hardware and software; they can be either static or mobile; they can be used for many different human activities, each with particular critical issues; network topologies are application-dependent, and as such, may present different criticalities and points of failure. Second, the environment where devices are deployed can also impact the reliability of the system: it may negatively affects sensed values, it could also cause intermittent connectivity, or it can even cause the devices to crash; furthermore, most of times devices are unattended, thus more prone to tampering. Finally, IoT devices typically communicate through wireless channels, which is more unreliable than wired communication. Wireless communication can be affected by potential interference caused by a harsh environment or by cyber attackers.

In this paper we review and analyze redundancy-based fault-tolerant techniques proposed so far for the IoT. We identify three complementary areas corresponding to the three main tasks performed by the nodes of an IoT network, that are sensing, routing, and control. We discuss how the implementation of fault-tolerance in each of these areas is primary for the correct operation of an entire system. We present an overview of the approaches used in sensing and routing. These two areas have fairly developed techniques. The area of control is less developed. Control devices can be seen as state machines and replication 
of the state machine as the redundancy for fault-tolerance [69]. Traditionally state-machine replication for fault-tolerance is realized through consensus protocols. Most of these protocols were developed in the 80's and 90's, and they are embedded in many distributed systems [13] [56]. We will present an overview of such protocols and discuss which are appropriate to support fault-tolerance within IoT. These algorithms though were not developed for environments where nodes participating in the protocol come in and go out of the network and will not cover very dynamic IoT scenarios. They could also take quadratic time with respect to the number of participants to terminate, limiting their scalability. We are, however, witnessing the emergence of a new class of consensus algorithms initially developed to support crypto-currencies [55] [76] and later extended to the more general abstraction of distributed ledgers [15] where contracts and other type of transactions can be implemented. We will review some recent results in these new class of algorithms and show how they can provide some of the flexibility required to support implementations of fault-tolerance in control devices in dynamic IoT scenarios.

\section{Preliminaries}

\subsection{The autonomic computing paradigm}

Autonomic systems (AS) are able to manage errors and failures, and dynamically adapt themselves to changes, without the need of human intervention. These features are increasingly important as the size and complexity of systems grow.

IoT systems are complex for their very nature as they are characterized by a high degree of heterogeneity and large number of components. Such heterogeneity raises the sources of errors and faults, expanding the attack surface, as well as increasing the difficulty of deploying all-encompassing security solutions. Hence, they should strive to attain safe autonomic governance.

The autonomic computing initiative promoted by IBM since the early 2000s [40] outlines four basic principles that characterize an autonomic system:

- self-configuring: self-configuring features allow a system for adapting dynamically to changes that occur into the environment where the system operates.

- self-healing: discovery, diagnosis, and reconfiguration are key tasks that help a system to be aware of the existence of faulty components in order to re-establish a coherent state after errors and components failures.

- self-optimizing: tuning resources and balancing workloads to maximize use of IT resources;

- self-protecting: anticipating, detecting, identifying, and protecting against attacks.

As we will see in the following sections, the satisfaction of one or more of above principles permits IoT systems to implement reliable routing, sensing, and control. Typically, fault-tolerant techniques are all self-protecting by definition, 
however most of them also implement one or more of the other three properties to be more robust against failures and attacks. In the next sections we will use these three tenets (self-configuring/healing/optimizing) as categories to classify the most significant fault-tolerant techniques found in the literature.

\section{$2.2 \quad$ Network model}

We consider a network model made of three different types of devices:

- sensor device: streams measurements to other devices;

- communication device: performs routing tasks, i.e. receives/forwards data packets from/to other devices, according to a routing protocol;

- control device: it takes decisions based on the measurement of sensors, it also may asks actuators to execute specific actions if required;

These three entities, along with actuators, are at the basis of the operation of various IoT scenarios, like cyber physical systems (CPS), industry 4.0, smart homes, smart cities, health care applications, etc. Sensors monitor physical phenomena and send sensed values to control devices directly or through communication devices; control devices take decisions on the basis of the received data. In many cases a single physical device may play multiple roles, e.g., a device can act as a sensor and as a router simultaneously. An example of an IoT application built according to our network model is depicted in Figure 1. In this scenario sensor devices detect the presence of cars and pedestrian, and send their readings to the control devices mounted on the semaphore to control its functioning. Notice that, sensor devices also work as communication devices.

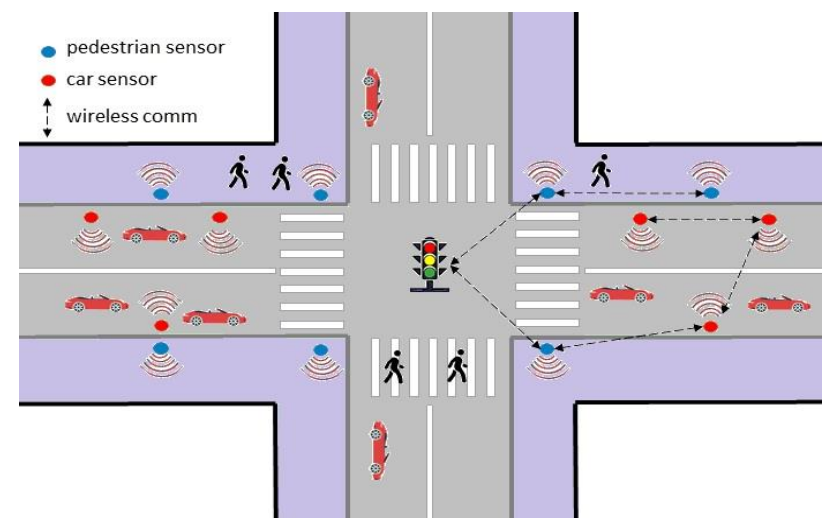

Fig. 1. A smart city application to control semaphores.

The accuracy of individual sensors readings is crucial in applications like surveillance network or health care IoT applications where the readings of sensors must be accurate to avoid false alarms and missed detection of important 
events. Fault-tolerance is achieved by (and its effectiveness is proportional to) the replication of the sensors, coupled with a fault detection task, or a sensor fusion algorithm (Section 3). In large scale networks, reliable routing is equally important as data travels along multi-hop routes. Fault-tolerant routing protocols replicate either the data or the path from the source to the destination (or both) to give data more chances to get to the destination (Section 4). Control devices deserve particular attention as if compromised they can take bad decisions that can lead to the crash of the system. Control devices can be replicated according to a state machine paradigm [69], where the replicas take decisions by running a distributed consensus algorithm [2] (Section 5). For each of these cases it's important to guarantee that faulty behavior of individual devices do not affect the overall operation of the system. ${ }^{4}$

We do not assume any particular network topology, network size, or IoT application, as we discuss the fault-tolerant techniques.

\subsection{Failure Classes}

Knowing how a device fails allows us to make realistic assumptions for implementing or choosing the most adequate algorithm to detect and handle failures. In the literature several classes of faults have been identified in relation with the device behavior. These classes fall into two general categories: fail-stop, that occurs when "in response to a failure, the component changes to a state that permits other components to detect that a failure has occurred and then stops" [68]; and byzantine, that occurs when "a component exhibits arbitrary or malicious behavior, perhaps involving collusion with other faulty components" [46].

Both fail-stop and byzantine faults may be caused by the actions of malicious users, that compromise a device to make it stop working, or to inject false data into the system. Fail-stop faults can also be caused by a harsh environment, by the total depletion of batteries, or by a hardware crash. However, it is not always possible to distinguish between a fail-stop fault and a byzantine behavior, especially in the absence of a diagnosis procedure. Thus, as byzantine faults do not put any constraints on how processes fail, a byzantine fault-tolerant algorithm can be extended to cover fail-stop faults.

\section{Fault-tolerant sensing}

Sensors may fail due to several reasons: the environment where they are deployed may negatively affect the sensed value or even causes the crash of a sensor; their unattended nature make them vulnerable to tampering; battery driven sensors may also stop working because they run out of energy supply. In such conditions, sensors are likely to not perform their work (fail-stop), or report unreliable readings (byzantine behavior) which would not reflect the true state

\footnotetext{
${ }^{4}$ Although IoT applications may comprise actuator devices also, we do not take them into account as the management of multiple actuators requires notions of electronics, a topic which fall out of the scope of this paper.
} 
of environmental phenomenon or events under monitoring. Sensor failures have been mainly addressed by means of redundancy, i.e., the replication of a service provided by a single device.

\subsection{Main approaches}

A basic approach to tolerate faulty sensors is to have several of them performing the same task and a centralized collector making multiple readings to decide the correct value by means of a fault-tolerant aggregation algorithm. One of the earliest results on how to deal with sensor failures was made by Marzullo [50]. The insight of his solution is the distinction the author makes between $a b$ stract and physical sensors. A physical sensor is a device used by a computer or controller to sample a physical variable; an abstract sensor is a piece-wise continuous function from a physical variable to a dense interval of physical values. In other words, an abstract sensor is an abstraction of a physical one. An abstract sensor is called correct if the interval estimate contains the actual value of the parameter being measured, otherwise it is called faulty. The abstraction of physical sensors in terms of intervals allows the interpolation of successive sensor readings (i.e., sensed values can be compared with each other) with known accuracy. In a situation where at most $f$ out of $n$ sensors are faulty, Marzullo considers all possible nonempty $(n-f)$ intersections of the $n$ sensors. A sensor which does not belong to any of the $(n-f)$ cliques is faulty since a correct sensor overlaps with at least $(n-f-1)$ other correct sensors. One and only one of the $(n-f)$ intersections contains the physical value. But since it is not possible to decide which intersection has the physical value, and since the output is required to be a connected interval, the smallest connected interval containing all the $(n-f)$ intersections is taken to be the output of the algorithm. The accuracy depends on the number of sensor replicas involved. Redundancy also provides protection against byzantine faults. The degree of sensor replication $n$ would be determined by the maximum number of tolerated faulty sensors $f$, and depending on the failure model we have: $(i)$ in applications where there exists a limit on the inaccuracy of a sensor (i.e., the width of the abstract sensor interval is finite), the system can tolerate at most $f=(n-1) / 2$ byzantine faults; $(i i)$ in applications where the inaccuracy of a sensor is unbounded, the system can tolerate at most $f=(n-1) / 3$ byzantine faults; (iii) in case of fail-stop faults only, the system can tolerate up to $f=n-1$ faulty nodes. These results are lifted from comparable results first obtained for consensus algorithms. We will present these results when we present replication of controllers in Section 5.1. From the point of view of autonomic management, Marzullos' approach results in self-protecting systems that can tolerate misbehaving sensors and can compute correct values without identifying the faulty sensors. However, if each interval is associated with a sensor identifier, a server may localize the source of the faults based on information such as the differences of physical intervals (sensors) values from the intersection chosen as the output interval and then use this new derived information to develop tools for self-healing capabilities. 
Since determining $n$ and, therefore, $f$ in advance might not be possible with full certainty, effort has been directed to doing more sophisticated analysis of historical sensor data, that can be used to get better correlation among the replicas as well as better detection of faulty sensors. Typically, iterative filtering (IF) algorithms are used to estimate some level of trustworthiness of a sensor based on the distance of its readings from the estimate of the correct values obtained in the previous iteration by some form of aggregation (usually a weighted average) of the readings of all sensors. Sensors whose readings significantly differ from such estimate are less trusted and, consequently, in the aggregation process of the current iteration their readings are given a lower weight. The performance of IF algorithms in the presence of different types of faults and false data injection attacks has been extensively studied showing significant improvements over simple averaging of values. The reader can find details of the basic approach in [20], and work addressing collusion attacks in [36] and [64].

These domain-independent approach can be extended with semantic information to improve the detection of faults. One type of semantic information is the relation between the geographical location of sensors and their readings. For example, sensing readings of acoustic volume and thermal radiation in sensor networks attenuate over distance. Such a piece of information can be used to differentiate between faulty readings and correct data. The authors of [29] leverage this concept and propose a fault-tolerant technique where faults are detected by assuming a mismatch between the distance rank and sensor data rank, i.e., a mismatch between the distance from the event and the sensed data.

An alternative approach to cope with faulty sensors is to make the collection of sensors that have been replicated to collect and report the same data agree in the sensing data to report. In other words, the sensors may implement a majority voting mechanism to decide what are the readings coming from faulty sensors, in order to avoid sending them to the collector. Two examples of such a distributed approach are [44] and [77]. In [44] the authors introduce the concept of spatial correlation, a piece of semantic information according to which readings from non-faulty sensors in close proximity are spatially correlated, i.e., similar because they are coming from adjacent places. With this in mind, a sensor can autonomously decide whether a neighbor is faulty or not. The author of [77] improved this mechanism by introducing a trustworthiness value for each sensor, such that the voting mechanisms is enhanced with the use of votes weighted according to the trust of sensor values. These techniques confer systems the property of being self-healing, as they would be able to autonomously detect and isolate faulty devices.

Another complementary approach to sensor replication is to have several sensors of different modalities be deployed to provide backup services for each other. More specifically, devices of different types may be deployed in order to have independent readings that can substitute each others in case of faults. For example, passive infrared sensors are often used to detect human presence. However, human presence can also be inferred by other types of sensors such as graphical monitors and sound sensors. This approach is discussed in [81], 
where the authors propose a self-healing technique which strength is directly proportional to the heterogeneity degree of the deployed devices.

Researchers have also looked at the problem of how many sensors to use and where best to locate them. The typical approach is to set the problem as an optimization problem that minimizes the number of sensors guaranteeing some minimal level of sensing coverage, so as if some sensor stops working, the sensing task continues to be performed correctly by its neighbors. Two examples of this line of work are [11] and [35], where the concept of $k$-coverage is used for ensuring a minimum quality of service. $k$-coverage is defined as any location in the network being monitored by at least $k$ nodes. The parameter $k$ measures the amount of fault-tolerance inherent in the sensors deployment scheme. These techniques only provide the self-protecting property, as the deployment of sensors is static, and no dynamic adjustments are expected in case of faults.

\section{Fault-tolerant routing protocols}

Data transmission in IoT is inherently faulty and unpredictable due to two main reasons. The first one is related to the brittle nature of wireless communication: links may fail when blocked by an external object or environmental condition, causing network partitions and changes in network topology. The second cause is linked to the failure of routers, that may stop working due to the harsh environment where they are deployed, or because of the depletion of batteries.

To these sources of faults we need to add malicious users compromising the communication channels and routers showing byzantine behavior as sources of faults. The action of an attacker aimed at preventing the use of a communication channel by interfering with the radio frequencies is known as jamming attack [54]. A router shows a byzantine behavior if it does not comply with the routing protocol rules. This typically happens when an attacker compromises a device by injecting malicious code by means of a malware. In literature, different types of byzantine behaviors have been identified in the field of wireless sensor networks [39], those that aim at disrupting the routing task are:

- dropping attack: malicious nodes do not forward part of (selective forwarding attack) or all (black hole attack) the received messages.

- replay attack: malicious nodes store received packets to forward them successively. This may be the cause of multiple issues: routing loops, extended or shortened source routes, generation of false error messages, network partition.

- delay attack: malicious nodes delay the forwarding of received packets so as to fail the time synchronization process, causing the production of excessive amount of routing control traffic.

- sybil attack: a malicious node presents multiple identities to other nodes in the network.

- sinkhole attack: malicious nodes lure network traffic by advertising better communication performance than their neighbors, thus creating a sinkhole with them in the center. The sinkhole attack occurs in protocols that use 
advertised information such as remaining energy or an estimate of end-to-end reliability to construct a routing topology.

\subsection{Main approaches}

Fault-tolerance in routing schemes is achieved by means of redundancy, which in turn, is implemented in various ways depending on the characteristics of the network. An effective and well established approach comes from the concept of dispersity routing introduced by Maxemchuk [51]. In dispersity routing mechanisms a source node divides the message and sends its components and possibly replicas of its components on different paths. This mechanism provides the ability of tolerating a large number of transmission errors before requiring the messages to be re-transmitted. This is the foundation of what is now referred to as multipath protocols. Multi-path approaches are mainly adopted in flat networks, i.e. where nodes are not organized hierarchically because all of them have more or less the same computing and communication capabilities, thus playing the same role. Dispersity based systems are classified as redundant or non-redundant. In a non-redundant system, a message is divided into a number of chunks equal to the number of paths between the source and the destination. Each chunk is sent on a different path and the message is then reconstructed at the destination. Faulttolerance is partially achieved since in case of link failure just a fraction of the original message has to be re-transmitted. In a redundant system the number of chunks is less than the number of paths. Additional chunks are formed as a linear combination of the bits in the message divisions, and each of the redundant and original chunks is transmitted along a different path. With an appropriate choice of linear combinations the message can be reconstructed without receiving all of the chunks. Intermittently connected networks, like mobile ad-hoc networks, vehicular ad-hoc networks and IoT applications deployed in harsh environments, have characteristics that made dispersity routing attractive. Messages routed over different paths whose nodes are completely disjoint withstand well dropping and sink hole attacks, as attackers should break at least one node for each path in order to be effective. However, completely disjoint paths may be difficult to create, especially in networks with a sparse topology. Braided paths (paths sharing some nodes but with no links in common) may be a viable alternative to provide probabilistic protection. For example, Ganesan et al. [27] proved that their braided multi-path scheme is a viable alternative for energy-efficient recovery from isolated and geographically correlated failures. Braided multi-paths require lower maintenance overhead than disjoint ones, and increasing the level of path disjointedness only provides modest gains in resilience gain with larger cost. Multi-paths have been used to extended standard protocols such as TCP [26] as well as bringing novel ideas for doing routing. For example, in the GRAB protocol proposed in [79], each node keeps the cost of forwarding a packet to the sink, such that nodes closer to the sink have smaller costs. Instead of a node choosing specific neighbors, each neighbor decides whether to forward a received packet by comparing its own cost to that of the sender. This way, data follow the direction of descending cost to reach the sink through multiple paths. Notice 
that the originality of this work lies in its ability to maintain multiple paths from the sources to the sink without the need to store them in routing tables.

Multi-path based approaches can also be implemented in a probabilistic way. In probabilistic broadcast approaches, called gossip protocols, a node randomly forwards packets to all its neighbors with a certain probability. Messages, then, follow multiple paths toward the destination, but unlike non-probabilistic protocols, such paths are not fixed but rather they are selected hop-by-hop. Since traffic is routed towards a physical location over multiple paths, it is difficult to create a sink hole type of attacks. Moreover, allowing nodes to choose the next hop using some probabilistic distribution, it reduces the chances of adversaries to successfully perform dropping attacks. The authors of [12] propose Anonymous Gossip, a probabilistic protocol that works in two concurrent phases. In the first phase a message is multicast, while in the second concurrent phase the gossip protocol recovers lost messages. Nodes need not know other nodes identity for gossip to be successful. This is extremely desirable for mobile nodes, that have limited resources, and where the knowledge of group membership is difficult to obtain. In contrast to the classical gossip algorithm where the same probability distribution to select the next hop is the same across all the nodes, the use of variable gossiping probabilities has been a common choice of many researchers, as it makes the work of an attacker more difficult. For example, in [4] the gossiping probability of a node is determined by the difference between its proximity to the destination and the proximity to the destination of the node from which the message was received. A comprehensive analysis on the choice of probabilities and protocols on the basis of the network topology can be found in [30]. The topologies considered are: (i) non-sparse networks (e.g. grids or dense topologies); (ii) sparse networks; (iii) sparse networks with the possibility to specify the gossip probability for a node having a number of neighbors under a certain threshold; and (iv) several topologies with the possibility for a node to know whether a message is dying out, so as to introduce a further copy of the message in the network (self-healing property).

Although multi-path approaches withstand well the presence of faulty nodes, they don't account for the energy consumption caused by the high number of messages transmitted. One of the most energy taxing task in many wireless devices is communicating with other devices. For IoT applications where energy saving is a primary maintenance goal, the solution is hierarchical routing. This approach aims to cluster nodes so that cluster heads can do some aggregation and reduction of data transmitted in order to save energy. This pattern is repeated to form a hierarchy of clusters with the uppermost level cluster nodes reporting directly to the sink. When the node density is high, hierarchical routing protocols tend to achieve much better performance because of less overhead, shorter average routing path, and quicker set-up procedures. Cluster heads are typically chosen based on their communication capabilities, or on their strategical location w.r.t. the rest of the network. Since these protocols use advertised information to construct a routing topology they are prone to sink hole attacks, as attackers use to advertise better communication performances to attract network traffic. Fur- 
thermore, once a compromised node get all the traffic it can perform black-hole attacks. Two approaches have been suggested to provide fail-tolerance to cluster head nodes: (i) by means of redundancy by introducing more than one cluster head; and (ii) by changing the set of cluster heads frequently enough to make it difficult for adversaries to choose the right nodes to compromise. Hao et al. [32] investigated the problem of cluster-head placement so that every node can communicate with at least two neighboring cluster heads. Thus, when a cluster head fails, a node can transfer itself to another cluster and transmit to an active cluster-head (self-healing property). Kuhn et al. [45] have improved this model by increasing the minimum number of cluster-heads a node can communicate with. They convert the node placement problem into a $k$ minimum dominating set ( $k$-MDS) problem. The goal of $k$-MDS is to find the minimal subset of cluster-heads so that every node can communicate with at least $k$ cluster-heads. However, these approaches have a drawback related to the battery depletion of cluster-heads, since their battery is drained much earlier than that of others nodes. Boukerche et al. [5] proposed a method to overcome this issue, according to which cluster-heads are dynamically chosen considering their residual energy (self-configuring property). This way, cluster-heads change over time and energy dissipation is uniformly distributed among the nodes. LEACH (Low-Energy Adaptive Clustering Hierarchy) [33], is another clustering-based protocol aimed at distributing energy consumption that utilizes randomized rotation of local cluster-heads. This feature simultaneously provides the model self-configuring and self-optimizing properties. LEACH is very comprehensive: it builds up clusters of sensor nodes based on their signal strength, uses localized coordination to enable scalability and robustness for dynamic networks, and incorporates data fusion into the routing protocol to reduce the amount of information that must be transmitted to the base station.

For the delay, replay, jamming, and sybil attacks, redundancy is not sufficient to make routing resilient, methods based on attack detection and/or authentication are needed instead. Some of the work in this research area are discussed in Section 6.

\section{Fault-tolerant control devices}

Control devices in an IoT network provide stateful services by performing computations as a reaction to interactions with other entities in the network. For example, a control device may receive events from motion detectors which the controller can analyze and then trigger actuators to turn cameras on. The cameras will send images back to the controller that must identify if there is a moving object, authenticate the object, decide whether to provide access to the object to protected areas, and decide to trigger another set of actuators to lock or unlock doors accordingly. Such a central role makes control devices essential for the correct system operation, thus it is paramount to keep them running appropriately. State machine replication can be considered the accepted paradigm to work with to provide fault-tolerance to stateful services [69]. Informally, the 
state machine replication is a distributed computing paradigm for implementing a fault-tolerant service by replicating a server and make sure that the behavior of the replicas under the same conditions are identical. Hence, if some of the servers fail the correct replicas can continue to provide the service. State machine replication protocols aim to implement a linearly ordered log abstraction that represent the trace of execution as if it were a single state machine. For that to happen, there are two properties that must be satisfies: (1) all servers must have the same view of the logs, and (2) whenever an event occurs in the system, it is incorporated quickly in the log. The first property is known as consistency and the second as liveliness. These protocols are referred as consensus protocols or algorithms since consensus must be reached by all replicas as to what to add to the log and when. Consensus protocols are widely used in highreliability computing systems and are applied in many distributed applications. We have already seen that some fault-tolerant mechanisms for sensor devices rely on consensus algorithms.

There are many situations in which many consensus algorithms can be simply adapted for IoT, but there are also scenarios for which the standard algorithms have limitations. In the next section we present brief overview of the formal characteristics of the different approached of reaching consensus and reflect on their application in the IoT context.

\subsection{Distributed consensus algorithms}

Given a group of $n$ processing units, the main property of a fault-tolerant consensus algorithm is the maximum number of processing unit that can fail and still guarantee a correct consensus. It is important to add that a consensus algorithm is expected to be implemented by a group of processing elements that are inherently asynchronous. These processing elements communicate by message passing and the communication channels may or may not be reliable. Therefore, the second property to establish is the total number of message bits needed. The first result that we mention is a well-known impossibility results that says that without synchronization a deterministic consensus is impossible even if only one participant crashes during the protocol [25]. This result, sometimes referred as the FLP impossibility result, means that a deterministic algorithm must include some form of synchronization and that the algorithm will run in rounds of communication. Lamport et al. [46] demonstrated that tolerating $f$ byzantine faults requires at least $n=3 f+1$ participants, while tolerating $f$ fail-stops requires at least $n=2 f+1$ participants. Although a few variations exist, basically it is accepted that about $f+O(f)$ rounds are needed and the message bits required are $O\left(f^{2}\right)$. Randomization in the algorithm, where some operations are the result of a random choice, lower the number of the rounds needed to $O(\log (n))$. The liveliness property for randomized protocols becomes "every correct process eventually knows about the log with probability 1" [8], while the consistency property becomes "every participant eventually decides a log entry with probability $1 "[73]$. 
For synchronicity, two assumptions are usually made in the algorithms. One is that communication is reliable with a fixed delay, and the computation capabilities of the processes are homogeneous. These assumptions limit the applicability in many IoT scenarios where networks might be unreliable and device capabilities heterogeneous. A typical approach to work around the FLP impossibility result is to work with a partial synchronous model [23], where upper bounds on message transmission time $\Delta$, and on process response time $\Phi$, can be established and time constraints can be violated only by system failures. According to this model, a system can behave asynchronously for some time interval until it eventually stabilizes and starts to behave in a synchronous manner, i.e. by respecting $\Delta$ or $\Phi$, or both. This time interval is called the global stabilization time (GST), it is unknown to the processors, but it is assumed that after the GST has elapsed the protocol will behave synchronously for a time interval long enough to achieve consensus. Algorithms based on this model are also probabilistic as they guarantee correctness only when these time constraints are satisfied. Various algorithms exist relaxing in different ways the assumptions, but the general result bounds the numbers of rounds to $O(n+\Delta+\Phi)$.

Another common approach to circumvent the FLP result is to extend an asynchronous system with an oracle. Oracles are typically failure detectors that detect, or suspect, when a replica crashes or behaves maliciously. The intuition behind oracles is that, having an idea about the failure/crash of a device, helps bypassing the FLP result as the impossibility derives from the impossibility of distinguishing faulty replicas from slow ones. Failure detectors for byzantine processors have the limitation that cannot detect all faults, but only a subset, and that they can not be completely independent of the consensus algorithm [42]. Chandra and Toueg [14] introduced the concept of unreliable failure detector, and showed how to use it to solve consensus in asynchronous systems with failstops. The term "unreliable" means that the detector can make mistakes, i.e. it can erroneously report that a process has crashed even though it is still running. The resilience of the approach is for $n=f+1$ processors, and termination happens after in $n-1$ rounds.

A way to limit the capabilities of byzantine replicas is to use authentication. Authentication is the ability of replicas to establish reliable communication channels to exchange messages. This limits the capabilities of faulty participants as: (i) a message signed by a fault-free replica is unable to be forged; (ii) any corruption of the message is detectable; and (iii) the signature can be authenticated by any other replica. As a consequence, authentication provides a system with a higher resilience. Furthermore, in authenticated systems there is no need for point-to-point private channels among replicas, and messages can also be sent through multi-hop paths involving more than one replica (although they can be faulty replicas too). One of the first contributions in this area was from Dolev and Reischuk [22], that proposed a deterministic consensus protocol for synchronous systems, which provides a resilience of $n=f+1$ processors, with termination happening after $O(f)$ rounds, employing $O\left(n+f^{2}\right)$ messages and $O(n f)$ signatures. An authenticated-byzantine fault detector was proposed by Kihlstrom et 


\begin{tabular}{|c|c|c|c|c|c|c|}
\hline Resilience & No. rounds & Time model & Fault & Det/Prob & Method & Ref \\
\hline$f \leq(n-1) / 3$ & $\geq f+1$ & synch & byzantine & det & - & [59] \\
\hline$O\left(f^{1.5}\right)$ & $\geq f+1$ & synch & byzantine & det & - & [18] \\
\hline$f \leq(n-1) / 3$ & $\geq 2 f+2$ & synch & byzantine & det & - & [74] \\
\hline$f \leq(n-1) / 3$ & $\leq f+1$ & asynch & byzantine & prob & randomization & [6] \\
\hline$f \leq n /(3+\epsilon)$ & $O(\log n)$ & synch & byzantine & prob & randomization & {$[7]$} \\
\hline$f \leq(n-1) / 3$ & $O(f / \log n)$ & synch & byzantine & prob & randomization & {$[17]$} \\
\hline$f<n-1$ & $O(f)$ & synch & auth. byzantine & det & - & {$[22]$} \\
\hline$f \leq(n-1) / 2$ & $\begin{array}{c}G S T+ \\
O(n+\Delta)\end{array}$ & $\begin{array}{l}\text { asynch comm. } \\
\& \text { synch proc. }\end{array}$ & $\begin{array}{c}\text { fail-stop } \\
\& \text { omission }\end{array}$ & prob & partially synch & [23] \\
\hline$f \leq n$ & $\begin{array}{c}G S T+ \\
O(n+\Delta+\Phi)\end{array}$ & $\begin{array}{l}\text { synch comm. } \\
\& \text { asynch proc. }\end{array}$ & fail-stop & prob & partially synch & {$[23]$} \\
\hline$f \leq(n-1) / 2$ & $\begin{array}{c}G S T+ \\
O(n+\Delta+\Phi)\end{array}$ & $\begin{array}{l}\text { asynch comm. } \\
\& \text { asynch proc. }\end{array}$ & $\begin{array}{c}\text { fail-stop } \\
\& \text { omission }\end{array}$ & prob & partially synch & {$[23]$} \\
\hline$f \leq(n-1) / 3$ & $\begin{array}{c}G S T+ \\
O(n+\Delta+\Phi)\end{array} \mid$ & $\begin{array}{l}\text { asynch comm. } \\
\text { \& asynch proc }\end{array}$ & $\begin{array}{c}\text { byzantine \& } \\
\text { auth. byzantine }\end{array}$ & prob & partially synch & {$[23]$} \\
\hline$f \in[(n-1) / 2, n / 2]$ & $\begin{array}{c}G S T+ \\
O(n+\Delta+\Phi)\end{array}$ & $\begin{array}{l}\text { synch comm. } \\
\& \text { asynch proc. }\end{array}$ & omission & prob & partially synch & {$[23]$} \\
\hline$f \leq(n-1) / 3$ & $\begin{array}{c}G S T+ \\
O(n+\Delta)\end{array}$ & $\begin{array}{l}\text { asynch comm. } \\
\& \text { synch proc. }\end{array}$ & $\begin{array}{c}\text { byzantine \& } \\
\text { auth. byzantine }\end{array}$ & prob & partially synch & {$[23]$} \\
\hline$f \leq(n-1) / 3$ & $\begin{array}{c}G S T+ \\
O(n+\Delta)\end{array}$ & $\begin{array}{c}\text { synch comm. } \\
\& \text { asynch proc. }\end{array}$ & byzantine & prob & partially synch & {$[23]$} \\
\hline$f \leq(n-1) / 2$ & $\begin{array}{c}G S T+ \\
O(n+\Delta)\end{array}$ & $\begin{array}{c}\text { synch comm. } \\
\& \text { asynch proc. }\end{array}$ & auth. byzantine & prob & partially synch & [23] \\
\hline$f \leq n-1$ & $\geq n-1$ & asynch & crash & prob & oracle & [14] \\
\hline$f \leq(n-1) / 3$ & $\geq f+1$ & synch & byzantine & det & oracle & {$[42]$} \\
\hline$f \leq(n-1) / 2$ & $O\left(n^{2}\right)$ & asynch & crash & det & partially synch & {$[47]$} \\
\hline
\end{tabular}

Table 1. Comparison of the consensus protocols discussed in Section 5.1

al. in [42], where the authors achieve the resilience of $n=3 f+1$ processors in asynchronous systems, with a deterministic approach that terminates within $f+1$ rounds employing $(f+1)(3 n+1)$ messages.

\subsection{Consensus protocols for IoT}

In IoT, consensus protocols designed with the byzantine fault model in mind (Section 2.3), and for systems assumed to be asynchronous, are preferable considered the uncertainty that characterizes IoT scenarios. In fact, these algorithms are able to deal with issues coming from both the faults and time domain, which makes them tolerant to adverse environments and considerable delays. However, there are limitations to these solutions. Table 1 summarizes the work discussed in this section so far. The reader can notice that these are well-established results coming from the 1990's and 80's. There are several limitations that hamper the utilization of these algorithms in IoT scenarios. One is that they all assume that the number of participants $n$ is fixed. This assumption might be reason- 
able in a smart home scenarios but it starts to create difficulties in more open situations as smart cities. A second limitation is that participants that don't respond to requested messages from the consensus protocols are pessimistically treated as malicious nodes, but in many IoT scenarios, no all honest nodes will be active all the time. Another potential difficulty can come from the number of messages or rounds required to complete the protocol. Most of the algorithms require a quadratic number of rounds or messages with respect to the number of participants. Examples of real cases where consensus has to be achieved among a non-fixed number of participants are in vehicular ad-hoc networks (VANET) $[34,31]$. Figure 2 shows a VANET scenario where a micro cloud is formed by smart cars, and where computation and decisions are performed among the cars that are in that region in the same time period. Another well fitting example is a set of unmanned air vehicles (UAV) or drones that patrol an area and that have to cooperate in unstable conditions like switching topology and time delay $[52]$.

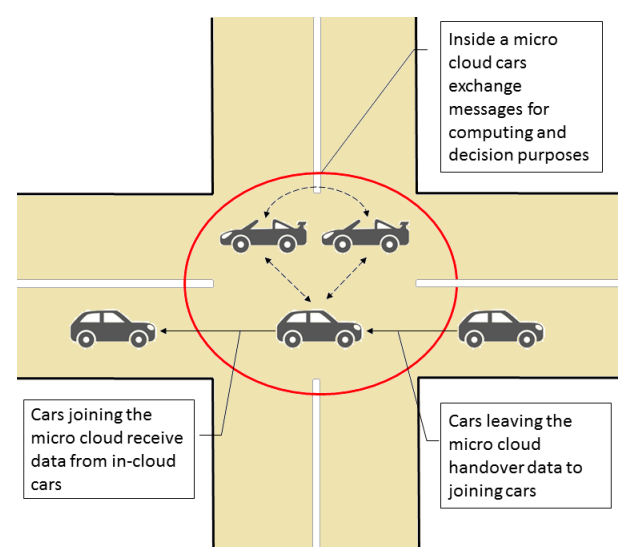

Fig. 2. A micro cloud in a vehicular ad-hoc network.

Since 2008, consensus algorithms took a new direction with the introduction of the concept of blockchain [55]. Informally, authentication has been used in consensus protocols to establish some level of trust among honest participants. In blockchain algorithms such trust is not established using cryptographic keys. Instead, trust is established by a "proof-of-work" (PoW) demonstrated through solving some computational puzzle that depends on the current event and the history of events. For that, each participant maintains a chain of blocks of events. Each block has a "key-less" signature that is obtained by the PoW. The first important contribution for the IoT is that consensus is reached with no more than a linear number of messages. The second relevant contribution is that by getting a copy of the most recent chain, nodes can enter into the system almost at any point. These consensus algorithms are the basis of all crypto-currencies generat- 
ing a lot of interest in the research community and industry. There has been a proliferation of blockchain protocols because of their potential impact on publicly verifiable transactions, and yet, able to provide strong privacy guarantees. There have been many suggestions about how blockchain algorithms can be useful to support IoT services focusing on the capabilities of supporting anonymous transactions. These suggestions tend to provide privacy or micro-payments based services [80]. Nevertheless, a fundamental application of consensus algorithms, state machine replication for fault-tolerance has never been mentioned. However, in order to incorporate the concept into consensus protocols for fault-tolerance in IoT the PoW must be replaced since it requires computational capabilities that the IoT devices are unlikely to have. This impediment is not limited to faulttolerance. Many of the applications summarized in [80] may not be practically realizable for the same reason. Recently though, we have seen technical results that are starting to address this issue. In [58], Pass and Shi propose an algorithm that significantly reduces the PoW by returning to strong authentications but keeping the blockchains. This access control layer allows honest participants to enter and leave the system with very few limitations. The authentication in the protocol requires from the participants some computational capacity, which may be not within the capabilities of all smart devices. However, smart devices computational power is increasing over time, thus in the near future they might be able to afford such heavier tasks. The characteristic of allowing participants to enter and leaving the system could be a key aspect for the implementation of distributed consensus in the IoT, which for some particular applications, it could result in a highly dynamic computing environment. For many consensus protocols the number of participants $n$ is a necessary information for processors to achieve consensus. With some little modification to the protocols, $n$ can be constrained in certain bounds, and the number of candidates participants be larger than $n$, thus leaving the protocol open to whoever holds the credentials to enter. This way, the system is conferred of a higher degree of resilience, as it can replace failed nodes in real time.

Besides PoW, Proof of Stake (PoS) is another mechanism to establish trust in blockchain protocols where the validator of the next transaction is chosen via a combinations of random selection and its reputation based on its performance over time in the system (stake) [41]. Here the "stake" is used as a measure of trust. PoS could be more suited for IoT application as it does not require high computational power to the participants, and can be exploited to model long-lived IoT devices, or devices more resilient to attacks and failures. There is another class of algorithms that can be characterized as combining standard Byzantine consensus with blockcain. In this class we find the Swirlds hash-graph consensus algorithm [1] and Algorand [28]. These algorithms are based on gossip protocols. These algorithm can significantly reduce the number of messages exchanged as well as the number of rounds to get consensus. Algorand also randomly selects the $n$ processes that will run the consensus and this set can be replaced in each round of the consensus protocol. The constraint is in the set of $n$ devices at least $2 n / 3$ are not faulty. 
Proving correctness and properties of distributed algorithms is not trivial. Many times the analysis is done under assumptions that are difficult or impossible to implement, and an independent verification and analysis is required [9]. This is the case of the algorithms discussed here: we are not aware of any implementation of the algorithm in [58], there are not publicly available implementations of the algorithm in [28], while the algorithm in [1] has an implementation but no independent validation.

\subsection{Beyond self-protecting autonomic management of faults}

A state machine as described so far has the characteristic of being self-protecting, a feature conferred by the employment of a consensus protocol which allows to withstand the presence of a minority of fail-stop or byzantine replicas. However it is desirable for a state machine to be self-healing too, so that crashed or compromised replicas can be identified and excluded from the consensus protocol.

As a brief example, let us consider an $f$-fault-tolerant state machine with $n=$ 19 participants running a consensus protocol which tolerates $f=(n-1) / 3=6$ faulty replicas. As long as the number of faulty replicas $t \leq f$ the state machine is capable of producing a correct output. When $t=f$ a non-self-healing state machine can not tolerate any more faults, thus as soon as $t$ exceeds $f$ the state machine might produce incorrect outputs for the next protocol executions. On the contrary, if the state machine is self-healing, i.e., it is able to discover and exclude faulty replicas, when $t$ is approaching $f$, it can update the value of $n$ to $n_{\text {new }} \leq n-t$. Let's say $t$ is 4 and $n_{n e w}=15$. The value of $f$ can be now $f_{\text {new }}=\left(n_{\text {new }}-1\right) / 3=4$. At this point the state machine can run the consensus protocol among the healthy replicas and tolerate 4 more faulty replicas, beside the 4 ones just excluded. Of course, it must be taken into account that a state machine with 14 replicas is less reliable than a state machine with 19 replicas. Removing faulty replicas also reduces both the message complexity and time complexity of the consensus protocols. A further improvement can be achieved if the state machine is able to replace faulty replicas with healthy ones, instead of just excluding them from the consensus protocol.

In blockchain-based consensus protocol, information about the state of the system can be found directly into the blockchain, since it contains the history of the events occurred within the system. The Algorand algorithm uses this to sidestep having to replace faulty replicas by replacing the consensus participants in every step, essentially reconfiguring the system all the time.

An important research direction is to find the most appropriate mechanisms to interleave these consensus protocols with the computation being done by the state machine to decide what part of the computation can be in practice considered atomic and become a block of the blockchain. In any case, the selfhealing property requires detecting the faulty controllers and then deciding how to restore their normal behavior. Detection is even more important in situations where the number of devices is not very large to randomly select $n$ controllers with more than $2 n / 3$ healthy controllers at every step. 
The methods for detection of faulty processes have been developed long time ago and it is typically performed by participants testing each others. A test consists of requesting the device to perform a computation and comparing the result with that which is expected. If the result disagrees with the expected answer, then the tested device is considered faulty [3, 43,62]. An effective method for testing is the remote attestation. Remote attestation allows for a tester to establish the absence of malicious changes to the memory contents of the tested replica. One way of performing remote attestation is to include an attestation routine in the replicas kernel that performs a checksum on the memory contents of the replica. As replicas are identical by definition, each replica knows the state of the other replicas' memory, thus each replica would also know the value of such a checksum at every moment $[70,71]$.

\section{Other research areas}

As mentioned in Section 4, for delay, replay, sybil, and jamming attacks there is no known redundancy based solution, rather, detection and/or prevention based mechanisms are more suitable. Delay attacks are typically addressed by means of intrusion detection mechanisms. The most common one is the watchdog, introduced by Marti et al. in [49]. According to this mechanism a network node is monitored by its neighbors, the watchdogs. Thus, if a node misbehaves it is detected by the watchdogs around it, and a failure tally is incremented. When the tally exceeds a certain threshold, a node is labeled as malicious and excluded from forwarding routes. The same approach has been extended so as watchdogs collaborate each other to investigate the source of intrusions and coordinate responses [37], and to be able to detect a wider range of attacks [72]. Some known protocols also implement security mechanisms against the delay attack, as for the RPL routing protocol for low power and lossy networks [75], which can be set to run in different security modes, each providing protection against different attacks.

For replay and sybil attacks we are aware of very few fault-tolerant technique able to withstand the presence of node showing such malicious behaviors $[19,21$, 57]. Rather, for these kind of attacks literature mainly provides prevention methods based on authentication. Authentication can prevent against replay attacks, because messages authenticated with previously disclosed keys are ignored. Many routing protocols also adopt sequence numbers in forwarded packets to protect against replay attacks, so as a packet with sequence number smaller than the last received one can be detected as a replayed packet. Authentication can also prevent sybil attacks, as neighbor nodes can use a cryptographic key to implement an authenticated communication channel, where identities are verified.

The jamming attack differs from the attacks discussed above because it is typically carried by an external attacker who does not need to compromise any network node to perform the attack. Moreover, this attack shows the same symptoms of any casual event that can interfere in the routing task (like changes in the environment), that is the unavailability of communication channels. In [10] 
the authors identify 12 types of "jammers" and propose two anomaly detection mechanisms to differentiate between legitimate and adversary scenarios. These mechanisms use three metrics to detect and differentiate jamming attacks from each other and from natural causes: the packet delivery ratio (PDR), the ratio of the number of delivered packets compared to the number of sent out packets; the bad packet ratio (BPR), the ratio of the number of erroneous packets compared to the number of received total or preamble packets for a sensor node; and the energy consumption amount (ECA), defined as approximated energy amount consumed in a specified time for a sensor node. The detection algorithms are loaded on network nodes, thus forming a distributed and localized detection system. A totally different approach is the spreading technique, whereby resilience to interference is achieved by transmitting information using a bandwidth much larger than its required minimum bandwidth [60]. In this approach, the transmitter redundantly encodes information using a spreading code. Then the receiver decodes the message by correlating the signal with the same code. Without knowing the spreading code used in the communication between two nodes, signals such as jamming or casual interference will appear noise-like upon decoding, and thus can be filtered out. However, if an attacker compromises a node, he/she can discover the spreading code in use and perform the attack successfully. To overcome such a circumstance Chiang et al. [16] proposed to adopt an asymmetric system, i.e. where sender and receiver use different codes, which allows network nodes to cooperatively detect a jamming attack.

In security, there are specialized tools for preventing (i.e., avoiding attacks) and monitoring (i.e., detecting attacks) faults in IoT. One of these tools is Kalis proposed by Midi et al. [53], an autonomic security tool for IoT networks. Kalis is self-configuring, as it discovers the features of the monitored network (size, topology, mobility, etc.) and enable the set of detection techniques that best fit with the sensed environment. Rullo et al. proposed PAST [65] a protocoladaptable security tool for the IoT. Like Kalis, PAST is self-configuring as it adapts its detection policy based on the set of communication protocols adopted by the monitored IoT application. PAST is self-optimizing too, as it leverages the security mechanisms of the protocols to optimize its detection task. Both Kalis and PAST can be integrated with recovery functions and replicated in order to implement a self-healing autonomic security system. A $k$-coverage-based method to compute the optimal replication degree and the optimal placement of security resources in IoT scenarios is presented in [66]. A similar approach for mobile IoT networks is presented in [67].

Fault-tolerance in IoT applications, especially in wireless sensor networks, has also been investigated in the area of the data storage. Not always sensors immediately forward sensed data to the sink, but rather they wait until the sink sends them a query. In this cases, as the failure of a sensor would result in a data loss, researchers have focused in designing failover techniques for data replications in order to optimally distribute sensed data over the entire network. An example of such work with local storage for replications is described in [63]. In this work, storage nodes are specified by hash functions to collect data of 
certain types. Redundancy is achieved by storing replicas directly on neighbors nodes. In [61], a dynamic replication for local data storage is presented. Here replicas are randomly distributed within a replication range influenced by the replica number and its density.

Beyond the IoT IoT networks normally do not have enough computing power to perform heavy tasks like computations on large amount of sensed data for control or analysis purposes. To this end, these tasks are typically delegated to more powerful computing infrastructures like the cloud and the fog. Faulttolerance approaches for the back-end support of IoT is beyond the scope of this paper, but there are fault detection and prevention mechanisms specifically addressing the potential harm that failures at the IoT can cause at the backend. In this context attackers may inject false data so as to compromise the outcome of the computation, or worse, to make issuing wrong control commands. Yan et al. [78] proposed a multi-level DDoS mitigation framework to defend against DDoS attacks for industrial IoT, which includes the fog and the cloud computing levels. Their approach implements firmware security checks, access control, malicious firmware/software detection, vulnerability scanning, intrusion detection, honeypot monitoring, and encryption of exchanged data. $\mathrm{Lu}$ et al [48] proposed a lightweight privacy preserving data aggregation (LPDA) scheme for fog computing-enhanced IoT. With the fog device deployed at the network edge, LPDA supports fault-tolerance by filtering false data injected by external attackers, and by computing mean and variance of the reported data even though some IoT devices are malfunctioning and stop reporting to the control center.

Cyber Physical Systems (CPS) share the same basic architecture with the IoT: they are typically made up of sensing and control components that communicate each other by means of some routing scheme. On the other hand, CPS may combine wired and wireless communication channels, they not necessarily consist of smart devices since they may have powerful computing components as integral part of the system. Controllers can complex and are mainly designed by means of control theory. Nevertheless, some basic principles to address faulttolerance in CPS are the same as in the IoT. In particular in the area of automatic control, fault detection and diagnosis methods as well as fault tolerant control designs have been developed in order to increase the reliability and maintainability of systems prone to failures. The results again resemble the results from consensus protocols, and similar to the IoT, fault-tolerant control systems have been designed so as to be self-protecting (called passive) and self-healing (called active). Although a detailed discussion of fault-tolerance in CPS is out of the scope of this paper, we refer the interested readers to [38] for a general overview and to [24] for the results on fault-tolerance in CPS control systems.

\section{Conclusion}

In this paper we have described redundancy-based fault-tolerant techniques for the IoT. We have divided the discussion in three parts, one for each role played 
by devices in IoT networks, namely, sensor, communication device, and control device. By analyzing the main contributions we have shown that the implementation of fault-tolerance in each of these areas is necessary for a system to be resilient to faults and failures. We have seen how to leverage redundancy to implement fault-tolerance, and what are the consequences in terms of trade-off between resilience and costs: fault-tolerance "is not for free", as redundancy implies more complexity in the operate and, consequently, more energy consumed and maintenance overhead. For sensors and routing protocols several fault-tolerant techniques have been proposed in literature: strategic sensors deployment, sensors fusion algorithms, centralized and distributed fault detection tasks, randomized routing protocols, etc. For control devices, instead, fault-tolerance has received less attention. We reviewed the possibility of implementing fault-tolerance with the state machine replication paradigm, and discussed different consensus protocols focusing on both classical and modern solutions. Thanks to recent proposals in the field of consensus protocols, we envision the possibility of making control in the IoT more dynamic, by allowing devices to implement distributed state machines that can change in size and capabilities over time.

\section{References}

1. Baird, L.: The swirlds hashgraph consensus algorithm: Fair, fast, byzantine fault tolerance. Swirlds, Inc. Technical Report SWIRLDS-TR-2016 1 (2016)

2. Barborak, M., Dahbura, A., Malek, M.: The consensus problem in fault-tolerant computing. ACM Computing Surveys (CSur) 25(2), 171-220 (1993)

3. Barsi, F., Grandoni, F., Maestrini, P.: A theory of diagnosability of digital systems. IEEE Transactions on Computers (6), 585-593 (1976)

4. Beraldi, R.: The polarized gossip protocol for path discovery in manets. Ad Hoc Networks 6(1), 79-91 (2008)

5. Boukerche, A., Pazzi, R.W.N., Araujo, R.B.: Fault-tolerant wireless sensor network routing protocols for the supervision of context-aware physical environments. Journal of Parallel and Distributed Computing 66(4), 586-599 (2006)

6. Bracha, G.: Asynchronous byzantine agreement protocols. Information and Computation 75(2), 130-143 (1987)

7. Bracha, G.: An o (log $\mathrm{n})$ expected rounds randomized byzantine generals protocol. Journal of the ACM (JACM) 34(4), 910-920 (1987)

8. Bracha, G., Toueg, S.: Resilient consensus protocols. In: Proceedings of the second annual ACM symposium on Principles of distributed computing. pp. 12-26. ACM (1983)

9. Cachin, C., Vukolić, M.: Blockchains consensus protocols in the wild. arXiv preprint arXiv:1707.01873 (2017)

10. Çakirolu, M., Özcerit, A.T.: Jamming detection mechanisms for wireless sensor networks. In: Proceedings of the 3rd international conference on Scalable information systems. p. 4. ICST (Institute for Computer Sciences, Social-Informatics and (2008)

11. Chakrabarty, K., Iyengar, S.S., Qi, H., Cho, E.: Grid coverage for surveillance and target location in distributed sensor networks. IEEE transactions on computers 51(12), 1448-1453 (2002) 
12. Chandra, R., Ramasubramanian, V., Birman, K.: Anonymous gossip: Improving multicast reliability in mobile ad-hoc networks. In: Distributed Computing Systems, 2001. 21st International Conference on. pp. 275-283. IEEE (2001)

13. Chandra, T.D., Griesemer, R., Redstone, J.: Paxos made live: an engineering perspective. In: Proceedings of the twenty-sixth annual ACM symposium on Principles of distributed computing. pp. 398-407. ACM (2007)

14. Chandra, T.D., Toueg, S.: Unreliable failure detectors for reliable distributed systems. Journal of the ACM (JACM) 43(2), 225-267 (1996)

15. Chen, J., Micali, S.: Algorand: the efficient and democratic ledger. CoRR abs/1607.01341 (2016)

16. Chiang, J.T., Hu, Y.C.: Cross-layer jamming detection and mitigation in wireless broadcast networks. In: Proceedings of the 13th annual ACM international conference on Mobile computing and networking. pp. 346-349. ACM (2007)

17. Chor, B., Coan, B.A.: A simple and efficient randomized byzantine agreement algorithm. IEEE Transactions on Software Engineering (6), 531-539 (1985)

18. Coan, B.A.: Efficient agreement using fault diagnosis. Distributed computing 7(2), 87-98 (1993)

19. Conti, M., Di Pietro, R., Mancini, L.V., Mei, A.: A randomized, efficient, and distributed protocol for the detection of node replication attacks in wireless sensor networks. In: Proceedings of the 8th ACM international symposium on Mobile ad hoc networking and computing. pp. 80-89. ACM (2007)

20. De Kerchove, C., Van Dooren, P.: Iterative filtering in reputation systems. SIAM Journal on Matrix Analysis and Applications 31(4), 1812-1834 (2010)

21. Demirbas, M., Song, Y.: An rssi-based scheme for sybil attack detection in wireless sensor networks. In: Proceedings of the 2006 International Symposium on on World of Wireless, Mobile and Multimedia Networks. pp. 564-570. IEEE Computer Society (2006)

22. Dolev, D., Reischuk, R.: Bounds on information exchange for byzantine agreement. Journal of the ACM (JACM) 32(1), 191-204 (1985)

23. Dwork, C., Lynch, N., Stockmeyer, L.: Consensus in the presence of partial synchrony. Journal of the ACM (JACM) 35(2), 288-323 (1988)

24. Fawzi, H., Tabuada, P., Diggavi, S.: Secure estimation and control for cyberphysical systems under adversarial attacks. IEEE Transactions on Automatic Control 59(6), 1454-1467 (2014)

25. FISCHER, M.J., LYNCH, N.A., PATERSON, M.S.: Impossibility of distributed consensus with one faulty process. Journal of the Assccktion for Computing Machinery 32(2), 374-382 (1985)

26. Ford, A., Raiciu, C., Handley, M., Barre, S., Iyengar, J.: Architectural guidelines for multipath tcp development. Tech. rep. (2011)

27. Ganesan, D., Govindan, R., Shenker, S., Estrin, D.: Highly-resilient, energyefficient multipath routing in wireless sensor networks. ACM SIGMOBILE Mobile Computing and Communications Review 5(4), 11-25 (2001)

28. Gilad, Y., Hemo, R., Micali, S., Vlachos, G., Zeldovich, N.: Algorand: Scaling byzantine agreements for cryptocurrencies. In: Proceedings of the 26th Symposium on Operating Systems Principles. pp. 51-68. ACM (2017)

29. Guo, S., Zhong, Z., He, T.: Find: faulty node detection for wireless sensor networks. In: Proceedings of the 7th ACM conference on embedded networked sensor systems. pp. 253-266. ACM (2009)

30. Haas, Z.J., Halpern, J.Y., Li, L.: Gossip-based ad hoc routing. IEEE/ACM Transactions on networking 14(3), 479-491 (2006) 
31. Hagenauer, F., Sommer, C., Higuchi, T., Altintas, O., Dressler, F.: Vehicular micro clouds as virtual edge servers for efficient data collection. In: Proceedings of the 2 nd ACM International Workshop on Smart, Autonomous, and Connected Vehicular Systems and Services. pp. 31-35. ACM (2017)

32. Hao, B., Tang, H., Xue, G.: Fault-tolerant relay node placement in wireless sensor networks: formulation and approximation. In: High Performance Switching and Routing, 2004. HPSR. 2004 Workshop on. pp. 246-250. IEEE (2004)

33. Heinzelman, W.R., Chandrakasan, A., Balakrishnan, H.: Energy-efficient communication protocol for wireless microsensor networks. In: System sciences, 2000. Proceedings of the 33rd annual Hawaii international conference on. pp. 10-pp. IEEE (2000)

34. Higuchi, T., Dressler, F., Altintas, O.: How to keep a vehicular micro cloud intact. In: 2018 IEEE 87th Vehicular Technology Conference (VTC Spring). pp. 1-5. IEEE (2018)

35. Hoblos, G., Staroswiecki, M., Aitouche, A.: Optimal design of fault tolerant sensor networks. In: Control Applications, 2000. Proceedings of the 2000 IEEE International Conference on. pp. 467-472. IEEE (2000)

36. Hoffman, K., Zage, D., Nita-Rotaru, C.: A survey of attack and defense techniques for reputation systems. ACM Computing Surveys (CSUR) 42(1), 1 (2009)

37. Huang, Y.a., Lee, W.: A cooperative intrusion detection system for ad hoc networks. In: Proceedings of the 1st ACM workshop on Security of ad hoc and sensor networks. pp. 135-147. ACM (2003)

38. Isermann, R.: Fault-diagnosis systems: an introduction from fault detection to fault tolerance. Springer Science \& Business Media (2006)

39. Karlof, C., Wagner, D.: Secure routing in wireless sensor networks: Attacks and countermeasures. In: Sensor Network Protocols and Applications, 2003. Proceedings of the First IEEE. 2003 IEEE International Workshop on. pp. 113-127. IEEE (2003)

40. Kephart, J.O., Chess, D.M.: The vision of autonomic computing. Computer (1), 41-50 (2003)

41. Kiayias, A., Russell, A., David, B., Oliynykov, R.: Ouroboros: A provably secure proof-of-stake blockchain protocol. In: Annual International Cryptology Conference. pp. 357-388. Springer (2017)

42. Kihlstrom, K.P., Moser, L.E., Melliar-Smith, P.M.: Byzantine fault detectors for solving consensus. The Computer Journal 46(1), 16-35 (2003)

43. Kreutzer, S., Hakimi, S.: Adaptive fault identification in two new diagnostic models. In: Proc. of the 21st Allerton Conference on Communication, Control and Computing. pp. 353-362 (1983)

44. Krishnamachari, B., Iyengar, S.: Distributed bayesian algorithms for fault-tolerant event region detection in wireless sensor networks. IEEE Transactions on Computers 53(3), 241-250 (2004)

45. Kuhn, F., Moscibroda, T., Wattenhofer, R.: Fault-tolerant clustering in ad hoc and sensor networks. In: null. p. 68. IEEE (2006)

46. Lamport, L., Shostak, R., Pease, M.: The byzantine generals problem. ACM Transactions on Programming Languages and Systems (TOPLAS) 4(3), 382-401 (1982)

47. Lamport, L., et al.: Paxos made simple. ACM Sigact News 32(4), 18-25 (2001)

48. Lu, R., Heung, K., Lashkari, A.H., Ghorbani, A.A.: A lightweight privacypreserving data aggregation scheme for fog computing-enhanced iot. IEEE Access 5, 3302-3312 (2017) 
49. Marti, S., Giuli, T.J., Lai, K., Baker, M.: Mitigating routing misbehavior in mobile ad hoc networks. In: Proceedings of the 6th annual international conference on Mobile computing and networking. pp. 255-265. ACM (2000)

50. Marzullo, K.: Tolerating failures of continuous-valued sensors. ACM Transactions on Computer Systems (TOCS) 8(4), 284-304 (1990)

51. Maxemchuk, N.F.: Dispersity routing. In: Proceedings of ICC. vol. 75 , pp. 41-10 (1975)

52. Maza, I., Kondak, K., Bernard, M., Ollero, A.: Multi-uav cooperation and control for load transportation and deployment. In: Selected papers from the 2nd International Symposium on UAVs, Reno, Nevada, USA June 8-10, 2009. pp. 417-449. Springer (2009)

53. Midi, D., Rullo, A., Mudgerikar, A., Bertino, E.: Kalisa system for knowledgedriven adaptable intrusion detection for the internet of things. In: Distributed Computing Systems (ICDCS), 2017 IEEE 37th International Conference on. pp. 656-666. IEEE (2017)

54. Mpitziopoulos, A., Gavalas, D., Konstantopoulos, C., Pantziou, G.: A survey on jamming attacks and countermeasures in wsns. IEEE Communications Surveys \& Tutorials 11(4) (2009)

55. Nakamoto, S.: Bitcoin: A peer-to-peer electronic cash system (2008)

56. Ongaro, D., Ousterhout, J.K.: In search of an understandable consensus algorithm. In: USENIX Annual Technical Conference. pp. 305-319 (2014)

57. Parno, B., Perrig, A., Gligor, V.: Distributed detection of node replication attacks in sensor networks. In: Security and Privacy, 2005 IEEE Symposium on. pp. 49-63. IEEE (2005)

58. Pass, R., Shi, E.: The sleepy model of consensus. In: International Conference on the Theory and Application of Cryptology and Information Security. pp. 380-409. Springer (2017)

59. Pease, M., Shostak, R., Lamport, L.: Reaching agreement in the presence of faults. Journal of the ACM (JACM) 27(2), 228-234 (1980)

60. Pickholtz, R., Schilling, D., Milstein, L.: Theory of spread-spectrum communications-a tutorial. IEEE transactions on Communications 30(5), 855-884 (1982)

61. Piotrowski, K., Langendoerfer, P., Peter, S.: tinydsm: A highly reliable cooperative data storage for wireless sensor networks. In: Collaborative Technologies and Systems, 2009. CTS'09. International Symposium on. pp. 225-232. IEEE (2009)

62. Preparata, F.P., Metze, G., Chien, R.T.: On the connection assignment problem of diagnosable systems. IEEE Transactions on Electronic Computers (6), 848-854 (1967)

63. Ratnasamy, S., Karp, B., Yin, L., Yu, F., Estrin, D., Govindan, R., Shenker, S.: Ght: a geographic hash table for data-centric storage. In: Proceedings of the 1st ACM international workshop on Wireless sensor networks and applications. pp. 78-87. ACM (2002)

64. Rezvani, M., Ignjatovic, A., Bertino, E., Jha, S.: Secure data aggregation technique for wireless sensor networks in the presence of collusion attacks. IEEE transactions on Dependable and Secure Computing 12(1), 98-110 (2015)

65. Rullo, A., Bertino, E., Saccà, D.: Past: Protocol-adaptable security tool for heterogeneous iot ecosystems. In: Dependable and Secure Computing, 2018 IEEE Conference on. pp. 46-53. IEEE (2018)

66. Rullo, A., Midi, D., Serra, E., Bertino, E.: Pareto optimal security resource allocation for internet of things. ACM Transactions on Privacy and Security (TOPS) $\mathbf{2 0}(4), 15(2017)$ 
67. Rullo, A., Serra, E., Bertino, E., Lobo, J.: Shortfall-based optimal placement of security resources for mobile iot scenarios. In: European Symposium on Research in Computer Security. pp. 419-436. Springer (2017)

68. Schneider, F.B.: Byzantine generals in action: Implementing fail-stop processors. Tech. rep., Cornell University (1983)

69. Schneider, F.B.: Implementing fault-tolerant services using the state machine approach: A tutorial. ACM Computing Surveys (CSUR) 22(4), 299-319 (1990)

70. Seshadri, A., Perrig, A., Van Doorn, L., Khosla, P.: Swatt: Software-based attestation for embedded devices. In: null. p. 272. IEEE (2004)

71. Shaneck, M., Mahadevan, K., Kher, V., Kim, Y.: Remote software-based attestation for wireless sensors. In: European Workshop on Security in Ad-hoc and Sensor Networks. pp. 27-41. Springer (2005)

72. da Silva, A.P.R., Martins, M.H., Rocha, B.P., Loureiro, A.A., Ruiz, L.B., Wong, H.C.: Decentralized intrusion detection in wireless sensor networks. In: Proceedings of the 1st ACM international workshop on Quality of service \& security in wireless and mobile networks. pp. 16-23. ACM (2005)

73. Toueg, S.: Randomized byzantine agreements. In: Proceedings of the third annual ACM symposium on Principles of distributed computing. pp. 163-178. ACM (1984)

74. Toueg, S., Perry, K.J., Srikanth, T.: Fast distributed agreement. SIAM Journal on Computing 16(3), 445-457 (1987)

75. Winter, T., Thubert, P., Brandt, A., Hui, J., Kelsey, R., Levis, P., Pister, K., Struik, R., Vasseur, J.P., Alexander, R.: Rpl: Ipv6 routing protocol for low-power and lossy networks. Tech. rep. (2012)

76. Wood, G.: Ethereum: A secure decentralised generalised transaction ledger. Ethereum project yellow paper 151, 1-32 (2014)

77. Xiao, X.Y., Peng, W.C., Hung, C.C., Lee, W.C.: Using sensorranks for in-network detection of faulty readings in wireless sensor networks. In: Proceedings of the 6th ACM international workshop on Data engineering for wireless and mobile access. pp. 1-8. ACM (2007)

78. Yan, Q., Huang, W., Luo, X., Gong, Q., Yu, F.R.: A multi-level ddos mitigation framework for the industrial internet of things. IEEE Communications Magazine 56(2), 30-36 (2018)

79. Ye, F., Zhong, G., Lu, S., Zhang, L.: Gradient broadcast: A robust data delivery protocol for large scale sensor networks. Wireless Networks 11(3), 285-298 (2005)

80. Yeow, K., Gani, A., Ahmad, R.W., Rodrigues, J.J., Ko, K.: Decentralized consensus for edge-centric internet of things: A review, taxonomy, and research issues. IEEE Access 6, 1513-1524 (2018)

81. Zhou, S., Lin, K.J., Na, J., Chuang, C.C., Shih, C.S.: Supporting service adaptation in fault tolerant internet of things. In: Service-Oriented Computing and Applications (SOCA), 2015 IEEE 8th International Conference on. pp. 65-72. IEEE (2015) 